\title{
Tabaquismo en Atención Primaria: Perfil de fumadoras consultantes, creencias y actitudes de los equipos de salud y oportunidades de intervención
}

\author{
Klaus Puschel I', Beti Thompson², Gloria Coronado², \\ Solange Rivera $\mathbf{M}^{1}$, Daniel Díaz $\mathbf{V}^{3}$, Loreto G onzález $\mathbf{V}^{1}$, \\ Genny Valencia $C^{1}$, Sibila Iñiguez $C^{1}$, Joaquín Montero $L^{1}$. \\ Smoking interventions in Primary \\ Health Care. Smoking profile of \\ women and beliefs and attitudes \\ of local health care teams
}

Background: Chile has one of the highest prevalence rate of smoking in the world. Brief counseling interventions for smoking cessation at the primary health care level are effective. Compliance with counseling intervention is strongly associated with beliefs and attitudes of the primary health care team that deliver it. The effectiveness of these interventions improve if they are applied to smoking populations with higher motivation of change and high self-efficacy for quitting. Aim: To study the smoking profile of a group of smoking women in Santiago and to identify beliefs and attitudes of the primary health care team members to implement smoking cessation interventions. Material and Methods: A cross-sectional design that included 306 women smokers attending two primary health care clinics in Santiago. Perceptions, beliefs and attitudes of 34 primary care team members from three clinics in Santiago were explored using a qualitative methodology. Results: The study identified a subgroup of $18 \%$ of women highly motivated to quit (decisional stage of change) and a $58 \%$ with a high self-efficacy. Beliefs and attitudes of staff at the clinics were characterized by invisibility, ambivalence and fatalism regarding the effectiveness of smoking cessation interventions. Conclusions: There is a subgroup of smoking women with a high probability of quitting if they receive an appropriate counseling. Developing a systematic approach for smoking cessation intervention at the primary care setting in Chile should consider the invisibility, ambivalence and fatalism of primary health care team members towards this topic (Rev Méd Chile 2006; 134: 726-34).

(Key words: Counseling; Primary health care; Smoking; Smoking cessation)

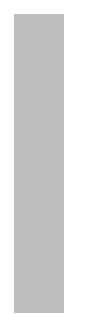

\begin{abstract}
Recibido el 12 de agosto, 2005. Aceptado el 7 de diciembre, 2005.
Este estudio ha sido financiado por el Instituto Nacional de Salud de EE.UU (NIH, RO3 TW005894-03) en colaboración con la Escuela de Medicina de la Pontificia Universidad Católica de Chile. El Instituto Nacional de EE.UU (NIH) no participó en el análisis, redacción o revisión del manuscrito. La colaboración con la Escuela de Medicina de la Pontificia Universidad Católica fue a través de la afiliación de algunos de los autores ${ }^{1}$ en calidad de académicos de dicha institución. ${ }^{1}$ Departamento de Medicina Familiar, Escuela de Medicina, Pontificia Universidad Católica de Chile. Santiago de Chile. ${ }^{2}$ Cancer Prevention Research Program, Fred Hutchinson Cancer Research Center, Seattle, WA. USA. ${ }^{3}$ Escuela de Psicología, Pontificia Universidad Católica de Chile.
\end{abstract}

Correspondencia a: Dr Klaus Puschel I. Edificio Decanato, Escuela de Medicina PUC. Lira 44, $1^{\text {er }}$ Piso, Santiago, Chile. Teléfonos: 3548111/3548688. Fax: 664 5408. E mail: kpuschel@med.puc.cl 
$\mathrm{C}$ hile es uno de los países con más alta prevalencia de tabaquismo a nivel mundial. Alrededor de $45 \%$ de los hombres y $40 \%$ de las mujeres en Chile son fumadores ${ }^{1,2}$. Esta prevalencia es casi el doble de la existente en países como Estados Unidos de Norteamérica (EE.UU) y es significativamente superior a $30 \%$ de la tasa de consumo ajustada observada a nivel mundial ${ }^{3}$. El gran aumento de la prevalencia de tabaquismo en Chile se produce en la población femenina. En los últimos 30 años, la prevalencia de tabaquismo sube desde $25 \%$ a $40 \%$ en las mujeres, mientras que se mantiene en alrededor de $45 \%$ en los hombres ${ }^{3}$.

Actualmente, en Chile el tabaco está vinculado con siete de las diez principales causas de muerte en la población ${ }^{4}$. El riesgo atribuible del tabaquismo con enfermedades cardiovasculares y cáncer se sitúa en alrededor de $20 \%$ a $30 \%^{5}$. En las mujeres, el tabaquismo tiene un especial impacto dado su asociación con enfermedades fetales, problemas del parto y enfermedades del niño ${ }^{5}$. En Chile, el tabaquismo genera un costo extra, descontando los ingresos por impuestos asociados a la venta del tabaco, para el estado de alrededor de US\$200 millones anuales ${ }^{6}$.

A nivel internacional, existe amplia evidencia que muestra que intervenciones sistemáticas en atención primaria, basadas en consejería breve y entrevista motivacional, pueden reducir la prevalencia de tabaquismo entre $3 \%$ y $30 \%$ anual $^{5,7}$. La variabilidad en la magnitud de este efecto está relacionada con la adherencia de los equipos de salud a la implementación de estas intervenciones. A su vez, el grado de adherencia a la consejería antitabaco depende en gran medida de la información, creencias y actitudes de los miembros del equipo de salud ${ }^{8-10}$.

En Chile no existe un modelo de intervención sistemático para enfrentar la gran población, especialmente en mujeres fumadoras consultantes que acuden a los centros de salud primario. Este estudio presenta información respecto al perfil de fumadoras consultantes en un grupo de centros de atención primaria en Santiago. El trabajo analiza las oportunidades de intervención y las percepciones, creencias y actitudes de tres equipos de salud respecto al tabaquismo y su enfrentamiento en la atención de salud.

\section{Metodología}

El estudio se realizó en 5 centros de Atención Primaria pertenecientes al área sur oriente de Santiago. En dos de estos centros (Bernardo Leighton, Puente Alto y San Rafael, La Pintana), se estudiaron las características de las fumadoras consultantes. En tres centros (El Roble, Santiago de Nuevo Extremo; La Pintana y Padre Villaseca, Puente Alto), se exploraron las percepciones, creencias y actitudes de los equipos de salud respecto al tabaquismo. La población promedio adscrita a los centros es de 45.000 personas y el promedio de funcionarios por centro es alrededor de 40 personas.

Muestreo. La selección de mujeres fumadoras consultantes en los centros de atención primaria se realizó en la sala de espera de cada uno de ellos. Durante dos meses (noviembre-diciembre), encuestadoras capacitadas abordaron a todas las mujeres consultantes de edad media, en la sala de espera de los centros participantes. Las mujeres fueron seleccionadas de acuerdo a su edad (18 a 50 años), hábito tabáquico (fumadoras de más de 100 cigarrillos en toda su vida y 10 más cigarrillos durante la última semana) y utilización del centro. Todas ellas otorgaron consentimiento informado escrito para participar en el estudio.

Para estudiar la percepción de los equipos de salud, se utilizó una metodología cualitativa en base a grupos focales. Se definió una muestra por conveniencia, mediante técnica de bola de nieve, en cada uno de los tres centros participantes. Los criterios de selección de la muestra fueron: presencia en cada grupo de profesionales, auxiliares paramédicos y administrativos, presencia de médicos y no médicos y participación de fumadores y no fumadores. Los invitados a participar fueron informados por escrito del estudio y del carácter voluntario de su participación. En total, se realizaron cuatro grupos focales con la participación de 34 personas.

Instrumentos. El perfil de fumadoras consultantes fue estudiado mediante la aplicación de una encuesta estructurada que contenía cinco secciones: datos demográficos, factores de riesgo y morbilidad asociada al tabaco, perfil de consumo (tipo y tiempo de consumo, adicción a nicotina), 
perfil de cambio de consumo (etapa de cambio, autoeficacia) y presencia de trastornos emocionales (escala de Goldberg) ${ }^{11}$.

El instrumento utilizado para valorar la etapa de cambio corresponde al diseñado por la Universidad de Rhode Island en Estados Unidos (URICA) ${ }^{12}$ y recomendada para su aplicación en atención primaria en Chile ${ }^{13}$. Mediante este instrumento se determina la etapa de cambio del paciente (precontemplativa, contemplativa, preparación) y por tanto la probabilidad de abandonar la conducta de riesgo $^{14}$. La autoeficacia se evaluó utilizando el instrumento desarrollado por la Universidad de New México, EE.UU y ampliamente utilizada a nivel mundial ${ }^{15}$. La autoeficacia evalúa la confianza que un paciente tiene de poder dejar el hábito tabáquico en caso de decidir hacerlo. El nivel de adicción a nicotina se estudió utilizando la escala de Fagerstrom abreviada, utilizada también en Chile ${ }^{16}$.

Registro de información cualitativa. El estudio cualitativo consideró la aplicación de dos pautas estructuradas. En la primera de ellas, se recogieron datos personales de los participantes y en la segunda, aplicada al final de la sesión, se les solicitó un resumen personal de los tópicos discutidos. La conducción de los grupos focales estuvo a cargo de dos profesionales (psicólogo y médico) con experiencia en metodología cualitativa y externos al centro de salud. Los investigadores utilizaron una pauta semiestructurada para abordar los tópicos del estudio. Cada grupo focal tuvo una duración aproximada de una hora.

La información obtenida en cada grupo focal fue grabada. Además, el investigador co-responsable registró, de acuerdo a una pauta previa, los contenidos discutidos y actitudes no verbales surgidas en la entrevista. Al final de cada entrevista ambos investigadores se reunieron por espacio de 30 min, para comparar percepciones y aclarar contenidos surgidos en la entrevista.

Análisis de la información. La información cuantitativa surgida de las entrevistas fue analizada utilizando el programa Epi-info versión 2000. Mediante este programa, se realizó el estudio estadístico descriptivo de la población participante.

El análisis de la información cualitativa se realizó utilizando un modelo abierto, axial y selectivo ${ }^{17}$. De esta forma, se realizó inicialmente una codificación abierta identificando eventos, términos o conceptos claves y equivalentes» que se agruparon en forma discreta. Posteriormente, se realizó un análisis axial en el cual los conceptos fueron categorizados y jerarquizados en base a sus condiciones, consecuencias e interrelaciones. Finalmente, se realizó el análisis selectivo de las categorías considerando los criterios de centralidad, frecuencia, consistencia y estabilidad ${ }^{17}$.

\section{RESUlTADos}

Para la caracterización de la población de fumadoras, se contactaron 320 mujeres consultantes, de ellas, 8 rechazaron participar en el estudio y en 6 se obtuvo información incompleta. En total, se incluyeron 306 mujeres fumadoras consultantes. La Tabla 1 muestra las características de la población de mujeres participantes. Puede observarse que se trata de una población de bajo nivel socioeconómico, predominantemente formada por dueñas de casa. Un porcentaje importante de este grupo de fumadoras presentaba trastornos de tipo emocional, enfermedades crónicas como hipertensión arterial (HTA) y diabetes mellitus (DM), 0 alguna morbilidad asociada al tabaco. Más de un tercio no se había realizado un Papanicolau en los últimos tres años.

La mayor parte de las mujeres estudiadas consumía menos de 15 cigarrillos al día, sin embargo, alrededor de $40 \%$ de ellas presentaba un alto grado de dependencia, de acuerdo a la escala de Fagerstrom. Dieciocho por ciento de las mujeres fumadoras se encontraba en una etapa de cambio avanzada, es decir, de preparación y un alto porcentaje de ellas reportó una alta autoeficacia para dejar de fumar.

En la Tabla 2 se muestra el perfil de participantes de los equipos de salud que integraron los grupos focales desarrollados. La mitad de los participantes fueron profesionales con una distribución homogénea entre médicos, enfermeras, matronas y otros (dentistas, nutricionistas, kinesiólogos). Alrededor de $60 \%$ de los participantes eran fumadores, predominando en este grupo los auxiliares y administrativos.

La Tabla 3 presenta un resumen de la información cualitativa analizada. En ella pueden obser- 
Tabla 1. Caracterización de la población y perfil de consumo de mujeres fumadoras consultantes

Características de la población y factores de riesgo $(n=306)$

Edad (años)

- Promedio (rango)

31,6

Ocupación (\%)

- Dueña de casa

- Asesora de hogar

- Servicios

Ingreso familiar mensual (pesos/mes, 2002) (\%)

- $<100.000$

- $101.000-250.000$

- $251.000-500.000$

- $>501.000$

Nivel educacional (años de educación) (\%)

- $0-4$

- 5-8

- $9-12$

- 13-14

- $>14$

Factores de riesgo (\%)

- HTA (tratamiento actual)

- DM (tratamiento actual)

- Sin PAP al día

- Trastorno emocional (GHQ >4)

Co-morbilidad (\%)

- Úlcera (tratamiento pasado o actual)

- Asma (tratamiento pasado o actual)

- Cardiopatía (tratamiento pasado o actual)

- Cáncer (tratamiento pasado o actual)

Perfil de consumo $(n=306)$

№ de cigarrillos/día

- Promedio ( ${ }^{\circ} \mathrm{de}$ cig/día)

- <15 cig/día (\%)

- $\geq 15 \mathrm{cig} /$ día (\%)

Edad de inicio de consumo (años)

- Promedio (DS)

Grado de dependencia (\%)

- Severa: Score Fagerstrom $>4$

Etapa motivacional de cambio (\%)

- Precontemplativa

- Contemplativa

- Preparación

Autoeficacia (\%)

- Muy alta

- Alta

- Baja

- Muy baja 
Tabla 2. C aracterísticas de la muestra de participantes de los equipos de salud

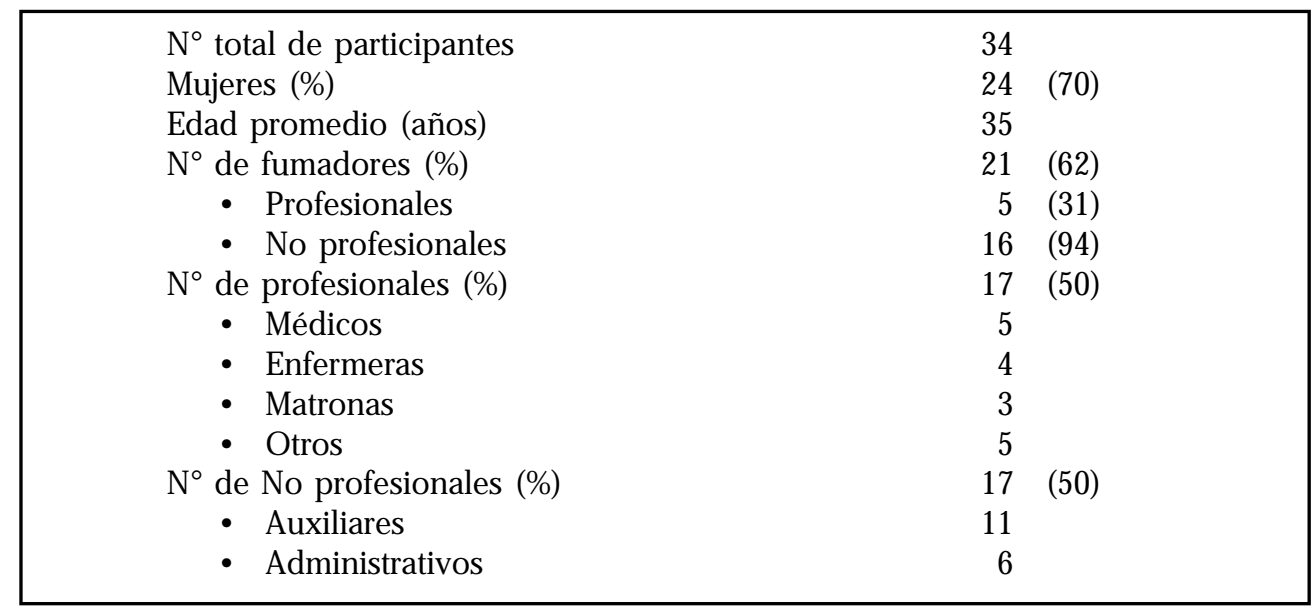

varse tres categorías esenciales: percepción del tabaquismo como problema de salud, actitud frente al hábito tabáquico y actitud frente a las estrategias para ayudar a los pacientes a dejar de fumar. En cada categoría resalta un tópico o concepto integrador y representativo. El tópico seleccionado requirió ser mencionado en todos los grupos desarrollados y, por al menos, la mitad de los participantes en cada grupo. Las citas presentadas ejemplifican el contenido discutido en torno a ese tópico de acuerdo al análisis de los investigadores. Los principales tópicos o conceptos identificados en los grupos fueron: invisibilidad, ambivalencia y fatalismo.

El concepto de invisibilidad se refiere a la falta de presencia 0 a la no identificación del tabaquismo como un problema de salud significativo. Una profesional participante reflejaba este concepto identificando el fumar «como una rutina», que distaba de ser un real problema, para ella fumar era «como lavarse los dientes». Un auxiliar paramédico manifestaba su escepticismo respecto a los efectos del tabaco al expresar que smucho de lo que se dice del cigarro es un mito».

La ambivalencia se vio expresada en forma consistente en la actitud discrepante de los grupos frente a intervenciones de consejería antitabáquica en la práctica asistencial. También se expresó en la tensión personal vs profesional respecto al tabaco. «o que sé, lo que creo y lo que hago con el tabaco no funciona todo alineado, todo es relativo y depende del momento y contexto en el que me encuentre». Esta ambivalencia se manifestó también en la percepción de lo soluntario»del hábito tabáquico. Por un lado fumar es una «pción personal», pero por otro produce una adicción tal que la «pción de dejarlo luego no resulta tan fácil».

El fatalismo representó una actitud consistente en los equipos de salud, al plantear el tema de las oportunidades y efectividad de las intervenciones antitabáquicas. Una profesional afirmaba «no conozco a nadie que siendo fumador empedernido y habiendo dejado de fumar, no haya engordado, todos recaen tarde o temprano». En todos los grupos se repitió la afirmación de que los programas y las campañas no sirven» Ningún grupo pudo mencionar alguna estrategia sistemática en el centro dirigida al tema tabaco, a diferencia de los programas de hipertensión o diabetes que fueron inmediatamente reconocidos por los participantes.

\section{DisCUSIÓN}

El tabaquismo constituye la causa de mortalidad prevenible más importante en Chile ${ }^{4}$. Existe clara evidencia sobre la eficacia de intervenciones preventivas para abandonar el tabaquismo en 
Tabla 3. Análisis axial de percepciones y creencias del equipo de salud respecto al tabaquismo

\begin{tabular}{|c|c|c|}
\hline Categorías & $\begin{array}{l}\text { Tópicos } \\
\text { principales }\end{array}$ & Contenidos: percepciones, creencias y actitudes \\
\hline $\begin{array}{l}\text { Percepción de } \\
\text { tabaquismo } \\
\text { como } \\
\text { problema de } \\
\text { salud }\end{array}$ & Invisibilidad & $\begin{array}{l}\text { - } \quad \text { Yo creo que mucho de lo que se dice del cigarro es un mito» } \\
\text { - «umar es un hábito, una rutina, como lavarse los dientes». } \\
\text { - «A diferencia del alcohol o las drogas que afectan a la familia, } \\
\text { el fumar lo afecta a uno, el que fuma se mata solo». } \\
\text { - «Todos pensamos lo mismo, el cigarro se asocia al descanso, } \\
\text { nos une, es nuestro punto de encuentro, sobre todo en el trabajo } \\
\text { que tenemos, la hora del cigarro, es parte de nuestra rutina». } \\
\text { - } \text { Es algo cultural, uno hace lo que tu sociedad te permita que } \\
\text { hagas, hay otros países donde fumar es casi un delito, acá es más } \\
\text { permitido, no es percibido como un real problema». }\end{array}$ \\
\hline $\begin{array}{l}\text { Percepción y } \\
\text { actitud frente } \\
\text { al tabaquismo }\end{array}$ & Ambivalencia & $\begin{array}{l}\text { - «umar es una opción, primero uno es persona, luego se } \\
\text { profesionaliza, muchos de los que fuman han empezado a fumar } \\
\text { antes de estudiar o trabajar y después ya no se puede dejar, pero } \\
\text { se respeta el espacio profesional». } \\
\text { - } \text { Lo que sé, lo que creo y lo que hago con el tabaco no funciona } \\
\text { todo alineado, todo es relativo y depende del momento y contexto } \\
\text { en el que me encuentre». } \\
\text { - «Fumar en el trabajo me hace sentir culpable, incoherente, pero } \\
\text { en mi casa... no tengo ningún problema... Fumar es un placer } \\
\text { culpable». } \\
\text { - Estar expuesto a los mismos problemas de salud, como el } \\
\text { tabaquismo, nos hace estar más cerca de los pacientes.... Lo } \\
\text { mejor sería que los programas antitabaco sean aplicados profe- } \\
\text { sionales y funcionarios no-fumadores». } \\
\text { - «Cómo darle a la gente razones para dejar de fumar si yo mismo } \\
\text { fumo?». } \\
\text { - } \text { एodos hablan de un ambiente libre de tabaco, pero en realidad } \\
\text { lo que se hace es resguardar un sitio físico para el fumador». }\end{array}$ \\
\hline $\begin{array}{l}\text { Actitud frente } \\
\text { a estrategias } \\
\text { para dejar de } \\
\text { fumar }\end{array}$ & Fatalismo & $\begin{array}{l}\text { - } \text { Dejar de fumar es muy difícil, sino imposible, al final todos re- } \\
\text { caen». } \\
\text { - «os programas y las campañas no sirven, sólo se deja de fumar } \\
\text { si alguna experiencia dramática cercana remece al fumador». } \\
\text { - «No hay conciencia en Chile de que el tabaco haga daño». } \\
\text { - ¿ualquier programa o campaña que tenga que ver con hábitos } \\
\text { tiene un pronóstico desfavorable, es casi imposible que pase } \\
\text { algo». } \\
\text { - } \text { ho conozco a nadie, que siendo fumador empedernido y } \\
\text { habiendo dejado de fumar, no haya engordado, todos recaen } \\
\text { tarde o temprano». }\end{array}$ \\
\hline
\end{tabular}


atención primaria. Como demuestra un metaanálisis que incluyó 43 estudios clínicos randomizados, la magnitud del efecto de consejería antitabaco está relacionada con el tipo de consejería, su intensidad y su diversidad ${ }^{18}$. De esta forma, la «calidad del consejo», «el número de acciones»y el abordaje «de equipo» logran una mayor eficacia que el consejo breve aislado ${ }^{18}$.

En relación a la calidad del consejo, se ha observado que éste aumenta su efectividad entre 2 y 4 veces, si considera la etapa de cambio y autoeficacia del paciente ${ }^{14,19,20}$. En nuestro estudio, 18\% de las mujeres fumadoras estaban en etapa de preparación. Este porcentaje es muy similar a $16 \%$ reportado en poblaciones de bajo nivel socioecónomico consultantes de atención primaria en EE.UU ${ }^{21}$, a $19 \%$ encontrado en una población similar en Holanda ${ }^{22}$ o a $16 \%$ encontrado en un estudio de más de 45.000 fumadores realizado en China ${ }^{23}$. De esta forma, el perfil de cambio de fumadoras consultantes en atención primaria en Chile, no parece diferir significativamente del de otras poblaciones a nivel mundial.

El alto grado de autoeficacia observado en nuestro estudio, así como el significativo porcentaje de mujeres en etapa de preparación, ofrecen oportunidades reales de desarrollar consejería antitabáquica eficaz en los centros de atención primaria estudiados.

En relación a la intensidad y diversidad de las intervenciones, es decir, al abordaje sistemático de equipo en la consejería antitabaco, la evidencia muestra que su aplicación está determinada en gran medida por las creencias y actitudes de los miembros del equipo de salud frente al tabaquis$\mathrm{mo}^{8,24,25}$

La invisibilidad encontrada en nuestro estudio, ya ha sido descrita en otras experiencias internacionales $^{26,27}$. En Chile, Cornejo y cols, describieron que alrededor de $50 \%$ de una muestra de 288 médicos en Santiago, no aconsejarían rutinariamente a pacientes fumadores asintomáticos que abandonaran su hábito. El problema aparecía, se hacía más visible, sólo en el grupo de pacientes sintomáticos ${ }^{28}$.

La ambivalencia identificada en nuestro trabajo, se ha observado también en diversos estudios que han mostrado el alto grado de valoración de los equipos de salud en la consejería antitabáquica pero su baja creencia de que pueda ser efectiva en su propia práctica ${ }^{24,26,29}$. La incapacidad de resolver la tensión personal vs profesional de los fumadores ha sido expresada por otros grupos como el «sentirse atrapado en el medio» de los roles personal y profesional ${ }^{30}$.

El fatalismo ha sido identificado en diversas experiencias como un factor predictor de baja adherencia a consejería por parte de los miembros del equipo de salud, independiente de factores organizacionales como carga asistencial y tiempo de atención ${ }^{8-10,31,32}$.

La nueva ley antitabaco, que se encuentra en su tramitación legislativa final ${ }^{33}$, aparece como una estrategia que puede favorecer una cultura menos permisiva para el tabaquismo en Chile y, de esta manera, impactar positivamente sobre las creencias y actitudes de los fumadores y de los equipos de salud.

Este estudio presenta algunas limitaciones que son importantes de comentar. En primer lugar, dado que el análisis cuantitativo se refiere a información de mujeres consultantes de dos centros de atención primaria en Santiago, no es posible generalizar la información obtenida a la población general, otros centros de salud, especialmente rurales, o a poblaciones masculinas. A pesar de esta limitación, es importante considerar que más de dos tercios de las consultantes en atención primaria son mujeres y que el aumento en la prevalencia de tabaquismo a nivel nacional, se asocia esencialmente a este grupo. El foco de este estudio corresponde precisamente a la población de mujeres consultantes en atención primaria, grupo en el cual, el equipo de salud tiene grandes oportunidades de realizar intervenciones preventivas.

En segundo lugar, es importante ser cautelosos a la hora de evaluar la validez externa de la información cualitativa obtenida de los equipos de salud. En metodología cualitativa, los resultados obtenidos no son "generalizables», dado que el muestreo realizado no es al azar sino por conveniencia. En el grupo de funcionarios estudiados, la prevalencia de tabaquismo fue mucho mayor (64\%) que la observada en la encuesta nacional de tabaquismo realizada a funcionarios de la salud en donde $40,7 \%$ de los funcionarios, en promedio, fue definido como fumador ${ }^{34}$. La técnica de muestreo utilizada en nuestro estudio (tipo sola de nieve»), buscó obtener la participación de 
personas que fueran representativas de las posturas y controversias existentes respecto al tópico a estudiar. Los criterios y metodología de selección utilizados fueron consistentes con este principio. Los resultados del estudio pueden ser transferibles $\gg 5$ a escenarios similares al estudiado.

En conclusión, este estudio presenta información relevante sobre el perfil de mujeres fumado-

\section{REFERENCIAS}

1. CONACE. Sexto estudio nacional de drogas en población general de Chile 2004 http:// www.conacedrogas.cl/inicio/pdf/Consumo cigarrillos_Chile_CONACEmayo2005.Pdf (Consultado 28 junio 2005).

2. MINSAL Departamento de Epidemiología. Encuesta nacional de salud, $2003 \mathrm{http}: / /$ epi.minsal. $\mathrm{cl} /$ epi/html/invest/ENS/ENS.htm (Consultado 6 de noviembre 2005).

3. CONACE. El consumo de cigarrillos en Chile. 1994-2004. Mayo 2004. http://www.conace drogas.cl/inicio/pdf/Consumo_cigarrillos_Chile CONACEmayo2005.Pdf (Consultado 28 junio 2005).

4. MINSAL Departamento de estadísticas e información en salud. Mortalidad por 10 primeras causas en Chile http://deis.minsal.cl/ev/mortalidad_general/causas/as.asp (Consultado 28 Junio 2005).

5. U.S. Preventive Services Task Force. Counseling to Prevent Tobacco Use and Tobacco-Related Diseases: Recommendation Statement. November 2003. Agency for Healthcare Research and Quality, Rockville, MD. (Consultado 28 junio 2005). http:// www .ahrq.gov/ clinic/3rduspstf/ to bacccoun/tobcounrs.htm

6. Consejo Nacional de Estupefacientes (CONACE). Ministerio del Interior Chile. Informativo CONACE. Informe especial sobre tabaco. http:// ww w.conacedrogas.cl/ inicio/noticias 2.php?d=1413 Enero 2005 (Consultado 28 junio 2005).

7. Whitlocka EC, Orleans CT, Penderc N, Aluand J. Evaluating primary care behavioral counseling interventions: An evidence-based approach. Am J Prev Med 2002; 22: 267-84. ras consultantes en atención primaria, identificando un subgrupo con altas probabilidades de cambio. El trabajo identifica un conjunto de creencias y actitudes de los equipos de salud esenciales de considerar para implementar estrategias de consejería antitabáquicas exitosas en el nivel primario de atención.

8. Orleans CT, George LK, Houpt JL, Brodie KH. Health promotion in primary care: A survey of US family practitioners. Prev Med 1985; 14: 636-47.

9. Kviz FJ, Clark MA, Prohaska TR. Attitudes and practices for smoking cessation counseling by provider type and patient age. Prev Med 1995; 24: 201-12.

10. Johnston JM, Chan SS, Chan SK, Lam TH, Chi I, LEUNG GM. Training nurses and social workers in smoking cessation counseling: a population needs assessment in Hong Kong. Prev Med 2005; 40: 389-406.

11. Araya R, Wynn R, Lewis G. Comparison of two self administered psychiatric questionnaires (GHQ-12 and SRQ-20) in primary care in Chile. Soc Psychiatry Psychiatr Epidemiol 1992; 27: 168-73.

12. Rustin TA. Assessing nicotine dependence. Am Fam Physician 2000; 62: 579-84, 591-2.

13. Instituto Nacional de Nutrición y Tecnología de Alimentos (INTA). Universidad de Chile. Modelos de Intervención Promocional en Atención Primaria http :/ w ww .inta.cl/ materialEducativo/Consejeriasenvidasana.pdf (Consultado 28 junio 2005).

14. ETTER JF, Sutton S. Assessing the stage of change in current and former smokers. Addiction 2002; 97: 1171-82.

15. Baer JS, Holt CS, Lichtenstein E. Self-efficacy and smoking reexamined: Construct validity and clinical utility. Journal of Consulting and Clinical Psychology 1986; 54: 846-52.

16. Sejuas D, Santander J, Ferrer V, Ramos AM. Dependencia de nicotina: seguimiento a un año plazo de pacientes tratados con terapia grupal más reemplazo de nicotina. Rev Méd Chile 1999; 127: 1313-20.

17. Strauss AL, Corbin J. Basics of qualitative research: Techniques and procedures for developing 
grounded theory. $2^{\text {nd }}$ Edition. 1998. Thousand Oaks, California: Sage.

18. US Public Health Service Report. A Clinical Practice Guideline for Treating Tobacco Use and Dependence. JAMA 2000; 283: 3244-54.

19. Spencer L, Pagell F, HaLion ME, Adams TB. Applying the transtheoretical model to tobacco cessation and prevention: a review of literature. Am J Health Promot 2002; 17: 7-71.

20. ETTER J. Associations between smoking prevalence, stages of change, cigarette consumption, and quit attempts across the United States. Prev Med 2004; 38: 369-73.

21. Gil KM, Schrop SL, Kune SC, Kimble EA, McCord G, McCormick KF, GILCHRIST VJ. Stages of change analysis of smokers attending clinics for the medically underserved. J Fam Pract 2002; 51: 1018.

22. Dotinga A, Schrijvers CT, Voorham AJ, Mackenbach JP. Correlates of stages of change of smoking among inhabitants of deprived neighbourhoods. Eur J Public Health 2005; 15: 152-9.

23. Yang G, Ma J, Chen A, Zhang Y, Samet JM, Taylor CE ET AL. Smoking cessation in China: findings from the 1996 national prevalence survey. Tob Control 2001; 10: 170-4.

24. Steptoe A. Attitudes to cardiovascular health promotion among GPs and practice nurses. Fam Pract 1999; 16: 158-63.

25. Coleman T, Wilson A. Anti-smoking advice from general practitioners: is a population-based approach to advice-giving feasible? $\mathrm{Br} \mathrm{J}$ Gen Pract 2000; 50: 1001-4.

26. Helgason AR, Lund KE. General practitioners' perceived barriers to smoking cessation-results from four Nordic countries. Scand J Public Health 2002; 30: 141-7.
27. Ohida T, SakURai $\mathrm{H}$, Mochizuki $\mathrm{Y}$, Kamal AM, TAKemura S, Minowa M. Smoking prevalence and attitudes toward smoking among Japanese physicians. JAMA 2001; 285: 2643-8.

28. Cornejo E, Medina E, Mascaro J, Matus P, Muñoz JC, CASTILO P. [Attitudes and behaviour concerning tobacco use among physicians from Santiago]. Rev Méd Chile 1994; 122: 1087-94.

29. KawaKami M, NaKamura S, Fumimoto H, Takizawa J, BABA M. Relation between smoking status of physicians and their enthusiasm to offer smoking cessation advice. Intern Med 1997; 36: 162-5.

30. Heath J, Andrews J, Keley FJ, Sorrell J. Caught in the middle: experiences of tobacco-dependent nurse practitioners. J Am Acad Nurse Pract 2004; 16: 396-401.

31. Braun BL, Fowles JB, Solberg LI, Kind EA, Lando $\mathrm{H}$, Pine D. Smoking-related attitudes and clinical practices of medical personnel in Minnesota. Am J Prev Med 2004; 27: 316-22.

32. Puschel K, Thompson B, Coronado G, López L, KIMBALL AM. Factors related to cancer screening in Hispanics: a comparison of the perceptions of Hispanic community members, health care providers, and representatives of organizations that serve Hispanics. Health Educ Behav 2001; 5: 57390.

33. CONACE. Noticias. Los cambios que plantea el proyecto de ley del tabaco http://www.conace drogas.cl/inicio/noticias2.php?d=1472 (Consultado 6 de noviembre de 2005).

34. Belo S, Soto M, Michaland S, Salnas J. Encuesta nacional de tabaquismo en funcionarios de salud. Rev Méd Chile 2004; 132: 223-32.

35. SOFAER SH. Qualitative research methods. Int J Qual Health Care 2002; 14: 329-36. 